\title{
Approximate Symmetries of the Harry Dym Equation
}

\author{
Mehdi Nadjafikhah and Parastoo Kabi-Nejad \\ School of Mathematics, Iran University of Science and Technology, Narmak, Tehran 1684613114, Iran \\ Correspondence should be addressed to Mehdi Nadjafikhah; m_nadjafikhah@iust.ac.ir
}

Received 27 October 2013; Accepted 17 November 2013

Academic Editors: B. Bagchi and Z. Qiao

Copyright (C) 2013 M. Nadjafikhah and P. Kabi-Nejad. This is an open access article distributed under the Creative Commons Attribution License, which permits unrestricted use, distribution, and reproduction in any medium, provided the original work is properly cited.

We derive the first-order approximate symmetries for the Harry Dym equation by the method of approximate transformation groups proposed by Baikov et al. $(1989,1996)$. Moreover, we investigate the structure of the Lie algebra of symmetries of the perturbed Harry Dym equation. We compute the one-dimensional optimal system of subalgebras as well as point out some approximately differential invariants with respect to the generators of Lie algebra and optimal system.

\section{Introduction}

The following nonlinear partial differential equation

$$
u_{t}=-\frac{1}{2} u^{3} u_{x x x}
$$

is known as the Harry Dym equation [1]. This equation was obtained by Harry Dym and Martin Kruskal as an evolution equation solvable by a spectral problem based on the string equation instead of Schrödinger equation. This result was reported in [2] and rediscovered independently in [3, 4]. The Harry Dym equation shares many of the properties typical of the soliton equations. It is a completely integrable equation $[5,6]$, which can be solved by inverse scattering transformation [7-9]. It has a bi-Hamiltonian structure and an infinite number of conservation laws and infinitely many symmetries $[10,11]$.

In this paper, we analyze the perturbed Harry Dym equation

$$
u_{t}+\frac{1}{2} u^{3} u_{x x x}+\varepsilon u_{x}=0
$$

where $\varepsilon$ is a small parameter, with a method which was first introduced by Baikov et al. [12, 13]. This method which is known as "approximate symmetry" is a combination of Lie group theory and perturbations. There is a second method which is also known as "approximate symmetry" due to
Fushchich and Shtelen [14] and later followed by Euler et al. $[15,16]$. For a comparison of these two methods, we refer the interested reader to $[17,18]$. Our paper is organized as follows. In Section 2, we present some definitions and theorems in the theory of approximate symmetry. In Section 3, we obtain the approximate symmetry of the perturbed Harry Dym equation. In Section 4, we discuss the structure of its Lie algebra. In Section 5, we construct the one-dimensional optimal system of subalgebras. In Section 6, we compute some approximately differential invariants with respect to the generators of Lie algebra and optimal system. In Section 7, we summarize our results.

\section{Notations and Definitions}

In this section, we will provide the background definitions and results in approximate symmetry that will be used along this paper. Much of it is stated as in [19]. If a function $f(x, \varepsilon)$ satisfies the condition

$$
\lim \frac{f(x, \varepsilon)}{\varepsilon^{p}}=0,
$$

it is written $f(x, \varepsilon)=o\left(\varepsilon^{p}\right)$ and $f$ is said to be of order less than $\varepsilon^{p}$. If

$$
f(x, \varepsilon)-g(x, \varepsilon)=o\left(\varepsilon^{p}\right),
$$


the functions $f$ and $g$ are said to be approximately equal (with an error $\left.o\left(\varepsilon^{p}\right)\right)$ and written as

$$
f(x, \varepsilon)=g(x, \varepsilon)+o\left(\varepsilon^{p}\right)
$$

or, briefly, $f \approx g$ when there is no ambiguity. The approximate equality defines an equivalence relation, and we join functions into equivalence classes by letting $f(x, \varepsilon)$ and $g(x, \varepsilon)$ be members of the same class if and only if $f \approx g$. Given a function $f(x, \varepsilon)$, let

$$
f_{o}(x)+\varepsilon f_{l}(x)+\cdots+\varepsilon^{p} f_{p}(x)
$$

be the approximating polynomial of degree $p$ in $\varepsilon$ obtained via the Taylor series expansion of $f(x, \varepsilon)$ in powers of $\varepsilon$ about $\varepsilon=0$. Then any function $g \approx f$ (in particular, the function $f$ itself) has the form

$$
g(x, \varepsilon)=f_{o}(x)+\varepsilon f_{l}(x)+\cdots+\varepsilon^{p} f_{p}(x)+o\left(\varepsilon^{p}\right) .
$$

Consequently the expression (6) is called a canonical representative of the equivalence class of functions containing $f$. Thus, the equivalence class of functions $g(x, \varepsilon) f(x, \varepsilon)$ is determined by the ordered set of $p+1$ functions $f_{0}(x), f_{l}(x), \ldots, f_{p}(x)$. In the theory of approximate transformation groups, one considers ordered sets of smooth vectorfunctions depending on $x$ 's and a group parameter $a$ :

$$
f_{0}(x, a), f_{l}(x, a), \ldots, f_{p}(x, a),
$$

with coordinates

$$
f_{0}^{i}(x, a), f_{1}^{i}(x, a), \ldots, f_{p}^{i}(x, a), \quad i=1, \ldots, n .
$$

Let us define the one-parameter family $G$ of approximate transformations

$$
\bar{x}^{i} \approx f_{0}^{i}(x, a)+\varepsilon f_{1}^{i}(x, a)+\cdots+\varepsilon^{p} f_{p}^{i}(x, a), \quad i=1, \ldots, n,
$$

of points $x=\left(x^{1}, \ldots, x^{n}\right) \in \mathbf{R}^{n}$ into points $\bar{x}=\left(\bar{x}^{1}, \ldots, \bar{x}^{n}\right) \in$ $\mathbf{R}^{n}$ as the class of invertible transformations

$$
\bar{x}=f(x, a, \epsilon),
$$

with vector-functions $f=\left(f^{1}, \ldots, f^{n}\right)$ such that

$$
\begin{aligned}
f^{i}(x, a, \epsilon) \approx & f_{0}^{i}(x, a)+\epsilon f_{1}^{i}(x, a) \\
& +\cdots+\varepsilon^{p} f_{p}^{i}(x, a), \quad i=1, \ldots, n .
\end{aligned}
$$

Here $a$ is a real parameter, and the following condition is imposed:

$$
f(x, 0, \epsilon) \approx x .
$$

Definition 1. The set of transformations (10) is called a oneparameter approximate transformation group if

$$
f(f(x, a, \varepsilon), b, \epsilon) \approx f(x, a+b, \varepsilon)
$$

for all transformations (11).
Definition 2. Let $G$ be a one-parameter approximate transformation group:

$$
\bar{z}^{i} \approx f(z, a, \varepsilon) \equiv f_{0}^{i}(z, a)+\varepsilon f_{1}^{i}(z, a), \quad i=1, \ldots, N .
$$

An approximate equation

$$
F(z, \varepsilon) \equiv F_{0}(z)+\varepsilon F_{1}(z) \approx 0
$$

is said to be approximately invariant with respect to $G$ or admits $G$ if

$$
F(\bar{z}, \varepsilon) \approx F(f(z, a, \varepsilon), \varepsilon)=o(\varepsilon)
$$

whenever $z=\left(z^{l}, \ldots, z^{N}\right)$ satisfies (16). If $z=\left(x, u, u_{(1)}, \ldots\right.$, $\left.u_{(k)}\right)$ then (16) becomes an approximate differential equation of order $k$, and $G$ is an approximate symmetry group of the differential equation.

Theorem 3. Equation (16) is approximately invariant under the approximate transformation group (15) with the generator

$$
X=X_{0}+\varepsilon X_{1} \equiv \xi_{0}^{i}(z) \frac{\partial}{\partial z^{i}}+\varepsilon \xi_{1}^{i} \frac{\partial}{\partial z^{i}},
$$

if and only if

$$
\left[X^{(k)} F(z, \varepsilon)\right]_{F \approx 0}=o(\varepsilon),
$$

or

$$
\left[X_{0}^{(k)} F_{0}(z)+\varepsilon\left(X_{1}^{(k)} F_{0}(z)+X_{0}^{(k)} F_{1}(z)\right)\right]_{(2.5)}=o(\varepsilon),
$$

where $X^{(k)}$ is the prolongation of $X$ of order $k$. The operator (18) satisfying (20) is called an infinitesimal approximate symmetry of or an approximate operator admitted by (16). Accordingly, (20) is termed the determining equation for approximate symmetries.

Theorem 4. If (16) admits an approximate transformation group with the generator $X=X_{0}+\varepsilon X_{1}$, where $X_{0} \neq 0$, then the operator

$$
X_{0}=\xi_{0}^{i}(z) \frac{\partial}{\partial z^{i}}
$$

is an exact symmetry of the equation

$$
F_{0}(z)=0 .
$$

Definition 5. Equations (22) and (16) are termed an unperturbed equation and a perturbed equation, respectively. Under the conditions of Theorem 4, the operator $X_{0}$ is called a stable symmetry of the unperturbed equation (22). The corresponding approximate symmetry generator $X=X_{0}+\varepsilon X_{1}$ for the perturbed equation (16) is called a deformation of the infinitesimal symmetry $X_{0}$ of (22) caused by the perturbation $\varepsilon F_{1}(z)$. In particular, if the most general symmetry Lie algebra of (22) is stable, we say that the perturbed equation (16) inherits the symmetries of the unperturbed equation. 


\section{Approximate Symmetries of the Perturbed Harry Dym Equation}

Consider the perturbed Harry Dym equation

$$
u_{t}+\frac{1}{2} u^{3} u_{x x x}+\varepsilon u_{x}=0 .
$$

By applying the method of approximate transformation groups, we provide the infinitesimal approximate symmetries (18) for the perturbed Harry Dym equation (2).

3.1. Exact Symmetries. Let us consider the approximate group generators in the form

$$
\begin{aligned}
X= & X_{0}+\varepsilon X_{1}=\left(\xi_{0}+\varepsilon \xi_{1}\right) \frac{\partial}{\partial x} \\
& +\left(\tau_{0}+\varepsilon \tau_{1}\right) \frac{\partial}{\partial t}+\left(\phi_{0}+\varepsilon \phi_{1}\right) \frac{\partial}{\partial u},
\end{aligned}
$$

where $\xi_{i}, \tau_{i}$, and $\phi_{i}$ for $i=0,1$ are unknown functions of $x, t$, and $u$. Solving the determining equation

$$
\left.X_{0}^{(3)}\left(u_{t}-\frac{1}{2} u^{3} u_{x x x}\right)\right|_{u_{t}-(1 / 2) u^{3} u_{x x x}=0}=0 \text {, }
$$

for the exact symmetries $X_{0}$ of the unperturbed equation, we obtain

$$
\begin{gathered}
\xi_{0}=\left(A_{1}+A_{2} x+\frac{A_{3}}{2} x^{2}\right), \\
\tau_{0}=\left(A_{4}+A_{5} t\right), \\
\phi_{0}=\left(A_{2}-\frac{1}{3 A_{5}}+x A_{3}\right) u,
\end{gathered}
$$

where $A_{1}, \ldots, A_{5}$ are arbitrary constants. Hence,

$$
\begin{aligned}
X_{0}= & \left(A_{1}+A_{2} x+\frac{A_{3}}{2} x^{2}\right) \frac{\partial}{\partial x} \\
& +\left(A_{4}+A_{5} t\right) \frac{\partial}{\partial t}+\left(\left(A_{2}-\frac{1}{3 A_{5}}+x A_{3}\right) u\right) \frac{\partial}{\partial u} .
\end{aligned}
$$

Therefore, the unperturbed Harry Dym equation admits the five-dimensional Lie algebra with the basis

$$
\begin{aligned}
& X_{0}^{1}=\frac{\partial}{\partial x}, \quad X_{0}^{2}=\frac{\partial}{\partial t}, \quad X_{0}^{3}=x \frac{\partial}{\partial x}+u \frac{\partial}{\partial u}, \\
& X_{0}^{4}=3 t \frac{\partial}{\partial t}-u \frac{\partial}{\partial u}, \quad X_{0}^{5}=x^{2} \frac{\partial}{\partial x}+2 x u \frac{\partial}{\partial u} .
\end{aligned}
$$

3.2. Approximate Symmetries. At first, we need to determine the auxiliary function $H$ by virtue of (19), (20), and (16), that is, by the equation

$$
H=\frac{1}{\varepsilon}\left[\left.X_{0}^{(k)}\left(F_{0}(z)+\varepsilon F_{1}(z)\right)\right|_{F_{0}(z)+\varepsilon F_{1}(z)=0}\right] .
$$

Substituting the expression (27) of the generator $X_{0}$ into (29) we obtain the auxiliary function

$$
H=u_{x}\left(A_{5}-A_{2}\right)+A_{3}\left(u-x u_{x}\right) .
$$

Now, calculate the operators $X_{1}$ by solving the inhomogeneous determining equation for deformations:

$$
\left.X_{1}^{(k)} F_{0}(z)\right|_{F_{0}(z)=0}+H=0
$$

So, the above determining equation for this equation is written as

$$
\begin{aligned}
& \left.X_{1}^{(3)}\left(u_{t}+\frac{1}{2} u^{3} u_{x x x}\right)\right|_{u_{t}+(1 / 2) u^{3} u_{x x x}=0} \\
& \quad+u_{x}\left(A_{5}-A_{2}\right)+A_{3}\left(u-x u_{x}\right)=0
\end{aligned}
$$

Solving the determining equation yields

$$
\begin{gathered}
\xi_{1}=\left(A_{5}-A_{2}\right) t-A_{3} x t+C_{4} x-C_{5}+\frac{C_{3}}{2} x^{2}, \\
\tau_{1}=\left(C_{1} t+C_{2}\right), \\
\phi_{1}=\left(-A_{3} t+C_{4}+C_{3} x+\frac{C_{1}}{3}\right) u,
\end{gathered}
$$

where $C_{1}, \ldots, C_{5}$ are arbitrary constants.

Thus, we derive the following approximate symmetries of the perturbed Harry Dym equation:

$$
\begin{gathered}
\mathbf{v}_{1}=\frac{\partial}{\partial x}, \quad \mathbf{v}_{2}=\frac{\partial}{\partial t}, \\
\mathbf{v}_{3}=x \frac{\partial}{\partial x}+u \frac{\partial}{\partial u}, \quad \mathbf{v}_{4}=3 t \frac{\partial}{\partial t}-u \frac{\partial}{\partial u} \\
\mathbf{v}_{5}=x^{2} \frac{\partial}{\partial x}+2 x u \frac{\partial}{\partial u}, \quad \mathbf{v}_{6}=\varepsilon \frac{\partial}{\partial x} \\
\mathbf{v}_{7}=\varepsilon \frac{\partial}{\partial t}, \quad \mathbf{v}_{8}=\varepsilon\left(x \frac{\partial}{\partial x}+u \frac{\partial}{\partial u}\right), \\
\mathbf{v}_{9}=\varepsilon\left(3 t \frac{\partial}{\partial t}-u \frac{\partial}{\partial u}\right), \quad \mathbf{v}_{10}=\varepsilon\left(x^{2} \frac{\partial}{\partial x}+2 x u \frac{\partial}{\partial u}\right) .
\end{gathered}
$$

Table 1 of commutators, evaluated in the first order of precision, shows that the operators (34) span a ten-dimensional approximate Lie algebra and hence generate a ten-parameter approximate transformation group.

Remark 6. Equations (34) show that all symmetries (28) of (1) are stable. Hence, the perturbed equation (2) inherits the symmetries of the unperturbed equation (1). 
TABLE 1: Approximate commutators of approximate symmetry of perturbed Harry Dym equation.

\begin{tabular}{ccccccccccc}
\hline & $\mathbf{v}_{\mathbf{1}}$ & $\mathbf{v}_{2}$ & $\mathbf{v}_{3}$ & $\mathbf{v}_{4}$ & $\mathbf{v}_{5}$ & $\mathbf{v}_{6}$ & $\mathbf{v}_{7}$ & $\mathbf{v}_{8}$ & $\mathbf{v}_{9}$ & $\mathbf{v}_{10}$ \\
\hline $\mathbf{v}_{\mathbf{1}}$ & 0 & 0 & $\mathbf{v}_{\mathbf{1}}$ & 0 & $2 \mathbf{v}_{3}$ & 0 & 0 & $\mathbf{v}_{6}$ & 0 \\
$\mathbf{v}_{2}$ & 0 & 0 & 0 & $12 \mathbf{v}_{2}$ & 0 & 0 & 0 & 0 & $3 \mathbf{v}_{7}$ & 0 \\
$\mathbf{v}_{3}$ & $-\mathbf{v}_{1}$ & 0 & 0 & 0 & $\mathbf{v}_{5}$ & $-\mathbf{v}_{6}$ & 0 & 0 & 0 & $\mathbf{v}_{10}$ \\
$\mathbf{v}_{4}$ & 0 & $-12 \mathbf{v}_{2}$ & 0 & 0 & 0 & 0 & $-3 \mathbf{v}_{7}$ & 0 & 0 \\
$\mathbf{v}_{5}$ & $-2 \mathbf{v}_{3}$ & 0 & $-\mathbf{v}_{5}$ & 0 & 0 & $-2 \mathbf{v}_{8}$ & 0 & $-\mathbf{v}_{10}$ & 0 \\
$\mathbf{v}_{6}$ & 0 & 0 & $\mathbf{v}_{6}$ & 0 & $2 \mathbf{v}_{8}$ & 0 & 0 & 0 & 0 \\
$\mathbf{v}_{7}$ & 0 & 0 & 0 & $3 \mathbf{v}_{7}$ & 0 & 0 & 0 & 0 & 0 \\
$\mathbf{v}_{8}$ & $-\mathbf{v}_{6}$ & 0 & 0 & 0 & $\mathbf{v}_{10}$ & 0 & 0 & 0 & 0 \\
$\mathbf{v}_{9}$ & 0 & $-3 \mathbf{v}_{7}$ & 0 & 0 & 0 & 0 & 0 & 0 & 0 \\
$\mathbf{v}_{10}$ & $-2 \mathbf{v}_{8}$ & 0 & $-\mathbf{v}_{10}$ & 0 & 0 & 0 & 0 & 0 & 0 \\
\hline
\end{tabular}

\section{The Structure of the Lie Algebra of Symmetries}

In this section, we determine the structure of the Lie algebra of symmetries of the perturbed Harry Dym equation. The Lie algebra $\mathbf{g}$ is nonsolvable, since

$$
\begin{gathered}
\mathbf{g}^{(1)}=[\mathbf{g}, \mathbf{g}]=\operatorname{Span}_{\mathbf{R}}\left\{\mathbf{v}_{1}, \mathbf{v}_{2}, \mathbf{v}_{3}, \mathbf{v}_{5}, \mathbf{v}_{6}, \mathbf{v}_{7}, \mathbf{v}_{8}, \mathbf{v}_{10}\right\} \\
\mathbf{g}^{(2)}=\left[\mathbf{g}^{(1)}, \mathbf{g}^{(1)}\right]=\operatorname{Span}_{\mathbf{R}}\left\{\mathbf{v}_{1}, \mathbf{v}_{3}, \mathbf{v}_{5}, \mathbf{v}_{6}, \mathbf{v}_{8}, \mathbf{v}_{10}\right\} \\
\mathbf{g}^{(3)}=\left[\mathbf{g}^{(2)}, \mathbf{g}^{(2)}\right]=\mathbf{g}^{(2)} .
\end{gathered}
$$

The Lie algebra $\mathbf{g}$ admits a Levi decomposition as the following semidirect product $\mathbf{g}=r \propto s$, where

$$
r=\operatorname{Span}_{\mathbf{R}}\left\{\mathbf{v}_{2}, \mathbf{v}_{4}, \mathbf{v}_{6}, \mathbf{v}_{7}, \mathbf{v}_{8}, \mathbf{v}_{9}, \mathbf{v}_{10}\right\}
$$

is the radical of $\mathbf{g}$ (the largest solvable ideal contained in $\mathbf{g}$ ) and

$$
s=\operatorname{Span}_{\mathbf{R}}\left\{\mathbf{v}_{1}, \mathbf{v}_{3}, \mathbf{v}_{5}\right\}
$$

is a semisimple subalgebra of $\mathbf{g}$.

The radical $r$ is solvable with the following chain of ideals:

$$
r^{(1)} \supset r^{(2)} \supset r^{(3)}=\{0\}
$$

where

$$
\begin{gathered}
r^{(1)}=\operatorname{Span}_{\mathbf{R}}\left\{\mathbf{v}_{2}, \mathbf{v}_{4}, \mathbf{v}_{6}, \mathbf{v}_{7}, \mathbf{v}_{8}, \mathbf{v}_{9}, \mathbf{v}_{10}\right\}, \\
r^{(2)}=\operatorname{Span}_{\mathbf{R}}\left\{\mathbf{v}_{2}, \mathbf{v}_{7}\right\} .
\end{gathered}
$$

The semisimple subalgebra $s$ of $\mathbf{g}$ is isomorphic to the Lie algebra $A_{3,8}$ of the classification of three-dimensional Lie algebras in [20], by the following isomorphism:

$$
\mathscr{T}:\left\{\mathbf{v}_{1}, \mathbf{v}_{3}, \mathbf{v}_{5}\right\} \longrightarrow\left\{\mathbf{v}_{1},-\mathbf{v}_{2},-\mathbf{v}_{3}\right\} .
$$

\section{Optimal System for Perturbed Harry Dym Equation}

Definition 7. Let $G$ be a Lie group. An optimal system of $s$-parameter subgroups is a list of conjugacy inequivalent $s$-parameter subgroups with the property that any other subgroup is conjugate to precisely one subgroup in the list. Similarly, a list of $s$-parameter subalgebras forms an optimal system if every $s$-parameter subalgebra of $\mathbf{g}$ is equivalent to a unique member of the list under some element of the adjoint representation: $\widetilde{\mathbf{h}}=\operatorname{Ad}(g(\mathbf{h})), g \in G$.

Proposition 8. Let $H$ and $\widetilde{H}$ be connected, s-dimensional Lie subgroups of the Lie group $G$ with corresponding Lie subalgebras $\mathbf{h}$ and $\widetilde{\mathbf{h}}$ of the Lie algebra $\mathbf{g}$ of $\mathrm{G}$. Then $\widetilde{\mathrm{H}}=\mathrm{gHg}^{-1}$ are conjugate subgroups if and only if $\widetilde{\mathbf{h}}=\operatorname{Ad}(g(\mathbf{h}))$ are conjugate subalgebras (Proposition 3.7 of [11]).

Actually, the proposition says that the problem of finding an optimal system of subgroups is equivalent to that of finding an optimal system of subalgebras. For one-dimensional subalgebras, this classification problem is essentially the same as the problem of classifying the orbits of the adjoint representation, since each one-dimensional subalgebra is determined by a nonzero vector in $\mathbf{g}$. To compute the adjoint representation one uses the Lie series:

$$
\begin{aligned}
\operatorname{Ad}\left(\exp \left(\mu \mathbf{v}_{i}\right)\right) \mathbf{v}_{j}= & \mathbf{v}_{j}-\mu\left[\mathbf{v}_{i}, \mathbf{v}_{j}\right] \\
& +\frac{\mu^{2}}{2}\left[\mathbf{v}_{i},\left[\mathbf{v}_{i}, \mathbf{v}_{j}\right]\right]-\cdots,
\end{aligned}
$$

where $\left[\mathbf{v}_{i}, \mathbf{v}_{j}\right], i, j=1, \ldots, 10$ is the commutator for the Lie algebra and $\mu$ is a parameter. In this manner, one constructs Table 2 with the $(i, j)$ th entry indicating $\operatorname{Ad}\left(\exp \left(\mu \mathbf{v}_{i}\right)\right) \mathbf{v}_{j}$.

Theorem 9. An optimal system of one-dimensional approximate Lie algebras of the perturbed Harry Dym equation is provided by

$$
\begin{gathered}
\mathbf{v}^{1}=\mathbf{v}_{8}, \\
\mathbf{v}^{2}=\mathbf{v}_{7}+a \mathbf{v}_{8}, \\
\mathbf{v}^{3}=\mathbf{v}_{6}+\mathbf{v}_{8}, \\
\mathbf{v}^{4}=\mathbf{v}_{6}-\mathbf{v}_{7}+\mathbf{v}_{8}, \\
\mathbf{v}^{5}=\mathbf{v}_{6}+\mathbf{v}_{7}+\mathbf{v}_{8}, \\
\mathbf{v}^{6}=\mathbf{v}_{2}+a \mathbf{v}_{8},
\end{gathered}
$$


TABLE 2: Adjoint representation of approximate symmetry of the perturbed Harry Dym equation.

\begin{tabular}{lccccccccccc}
\hline Ad & $\mathbf{v}_{1}$ & $\mathbf{v}_{2}$ & $\mathbf{v}_{3}$ & $\mathbf{v}_{4}$ & $\mathbf{v}_{5}$ & $\mathbf{v}_{6}$ & $\mathbf{v}_{7}$ & $\mathbf{v}_{8}$ & $\mathbf{v}_{9}$ & $\mathbf{v}_{10}$ \\
\hline $\mathbf{v}_{1}$ & $\mathbf{v}_{1}$ & $\mathbf{v}_{2}$ & $\mathbf{v}_{3}-\mu \mathbf{v}_{1}$ & $\mathbf{v}_{4}$ & $\mathbf{v}_{5}-2 \mu \mathbf{v}_{3}+\mu^{2} \mathbf{v}_{1}$ & $\mathbf{v}_{6}$ & $\mathbf{v}_{7}$ & $\mathbf{v}_{8}-\mu \mathbf{v}_{6}$ & $\mathbf{v}_{9}$ & $\mathbf{v}_{10}-2 \mu \mathbf{v}_{8}+\mu^{2} \mathbf{v}_{6}$ \\
$\mathbf{v}_{2}$ & $\mathbf{v}_{1}$ & $\mathbf{v}_{2}$ & $\mathbf{v}_{3}$ & $\mathbf{v}_{4}-12 \mu \mathbf{v}_{2}$ & $\mathbf{v}_{5}$ & $\mathbf{v}_{6}$ & $\mathbf{v}_{7}$ & $\mathbf{v}_{8}$ & $\mathbf{v}_{9}-3 \mu \mathbf{v}_{7}$ & $\mathbf{v}_{10}$ \\
$\mathbf{v}_{3}$ & $e^{\mu} \mathbf{v}_{1}$ & $\mathbf{v}_{2}$ & $\mathbf{v}_{3}$ & $\mathbf{v}_{4}$ & $e^{-\mu} \mathbf{v}_{5}$ & $e^{\mu} \mathbf{v}_{6}$ & $\mathbf{v}_{7}$ & $\mathbf{v}_{8}$ & $\mathbf{v}_{9}$ & $e^{-\mu} \mathbf{v}_{10}$ \\
$\mathbf{v}_{4}$ & $\mathbf{v}_{1}$ & $e^{12 \mu} \mathbf{v}_{2}$ & $\mathbf{v}_{3}$ & $\mathbf{v}_{4}$ & $\mathbf{v}_{5}$ & $\mathbf{v}_{6}$ & $e^{3 \mu} \mathbf{v}_{7}$ & $\mathbf{v}_{8}$ & $\mathbf{v}_{9}$ & $\mathbf{v}_{10}$ \\
$\mathbf{v}_{5} \mathbf{v}_{1}+2 \mu \mathbf{v}_{3}+\mu^{2} \mathbf{v}_{5}$ & $\mathbf{v}_{2}$ & $\mathbf{v}_{3}+\mu \mathbf{v}_{5}$ & $\mathbf{v}_{4}$ & $\mathbf{v}_{5}$ & $\mathbf{v}_{6}+2 \mu \mathbf{v}_{8}+\mu^{2} \mathbf{v}_{10}$ & $\mathbf{v}_{7}$ & $\mathbf{v}_{8}+\mu+\mathbf{v}_{10}$ & $\mathbf{v}_{9}$ & $\mathbf{v}_{10}$ \\
$\mathbf{v}_{6}$ & $\mathbf{v}_{1}$ & $\mathbf{v}_{2}$ & $\mathbf{v}_{3}-\mu \mathbf{v}_{6}$ & $\mathbf{v}_{4}$ & $\mathbf{v}_{5}-2 \mu \mathbf{v}_{8}$ & $\mathbf{v}_{6}$ & $\mathbf{v}_{7}$ & $\mathbf{v}_{8}$ & $\mathbf{v}_{9}$ & $\mathbf{v}_{10}$ & $\mathbf{v}_{8}$ \\
$\mathbf{v}_{7}$ & $\mathbf{v}_{1}$ & $\mathbf{v}_{2}$ & $\mathbf{v}_{3}$ & $\mathbf{v}_{4}-3 \mu \mathbf{v}_{7}$ & $\mathbf{v}_{5}$ & $\mathbf{v}_{6}$ & $\mathbf{v}_{7}$ & $\mathbf{v}_{8}$ & $\mathbf{v}_{9}$ & $\mathbf{v}_{10}$ \\
$\mathbf{v}_{8}$ & $\mathbf{v}_{1}+\mu \mathbf{v}_{6}$ & $\mathbf{v}_{2}$ & $\mathbf{v}_{3}$ & $\mathbf{v}_{4}$ & $\mathbf{v}_{5}-\mu \mathbf{v}_{10}$ & $\mathbf{v}_{6}$ & $\mathbf{v}_{7}$ & $\mathbf{v}_{8}$ & $\mathbf{v}_{9}$ & $\mathbf{v}_{10}$ \\
$\mathbf{v}_{9}$ & $\mathbf{v}_{1}$ & $\mathbf{v}_{2}+3 \mu \mathbf{v}_{7}$ & $\mathbf{v}_{3}$ & $\mathbf{v}_{4}$ & $\mathbf{v}_{5}$ & $\mathbf{v}_{6}$ & $\mathbf{v}_{7}$ & $\mathbf{v}_{8}$ & $\mathbf{v}_{9}$ & $\mathbf{v}_{10}$ \\
$\mathbf{v}_{10}$ & $\mathbf{v}_{1}+2 \mu \mathbf{v}_{8}$ & $\mathbf{v}_{2}$ & $\mathbf{v}_{3}+\mu \mathbf{v}_{10}$ & $\mathbf{v}_{4}$ & $\mathbf{v}_{5}$ & $\mathbf{v}_{6}$ & $\mathbf{v}_{7}$ & $\mathbf{v}_{8}$ & $\mathbf{v}_{9}$ & $\mathbf{v}_{10}$ \\
\hline
\end{tabular}

$$
\begin{gathered}
\mathbf{v}^{7}=\mathbf{v}_{2}-\mathbf{v}_{6}+a \mathbf{v}_{8}, \\
\mathbf{v}^{8}=\mathbf{v}_{2}+\mathbf{v}_{6}+a \mathbf{v}_{8}, \\
\mathbf{v}^{9}=\mathbf{v}_{1}+a \mathbf{v}_{2}+b \mathbf{v}_{7}, \\
\mathbf{v}^{10}=a \mathbf{v}_{1}+b \mathbf{v}_{2}+\mathbf{v}_{5}+c \mathbf{v}_{6}+d \mathbf{v}_{7}, \\
\mathbf{v}^{11}=a \mathbf{v}_{1}+b \mathbf{v}_{2}+\mathbf{v}_{3}+c \mathbf{v}_{5}+d \mathbf{v}_{7}+e \mathbf{v}_{8}, \\
\mathbf{v}^{13}=a \mathbf{v}_{1}+b \mathbf{v}_{3}+\mathbf{v}_{4}+c \mathbf{v}_{5}+d \mathbf{v}_{6}+e \mathbf{v}_{8}, \\
\mathbf{v}^{14}=a \mathbf{v}_{1}+b \mathbf{v}_{3}+c \mathbf{v}_{4}+d \mathbf{v}_{5}+e \mathbf{v}_{6}+f \mathbf{v}_{8}+\mathbf{v}_{9}, \\
\mathbf{v}^{15}=a \mathbf{v}_{1}+d \mathbf{v}_{5}+e \mathbf{v}_{6}+f \mathbf{v}_{8}+\mathbf{v}_{9}+b \mathbf{v}_{3}+c \mathbf{v}_{4}+d \mathbf{v}_{5}+e \mathbf{v}_{6}+f \mathbf{v}_{8}+\mathbf{v}_{9}, \\
\mathbf{v}^{16}=a \mathbf{v}_{1}+\mathbf{v}_{2}+b \mathbf{v}_{3}+c \mathbf{v}_{4}+d \mathbf{v}_{5}+e \mathbf{v}_{6}+f \mathbf{v}_{8}+\mathbf{v}_{9} .
\end{gathered}
$$

Proof. Consider the approximate symmetry algebra $\mathbf{g}$ of the unperturbed Harry Dym equation, whose adjoint representation was determined in Table 2. Given a nonzero vector

$$
\mathbf{v}=\sum_{i=1}^{10} a_{i} \mathbf{v}_{\mathbf{i}}
$$

our task is to simplify as many of the coefficients $a_{i}$ as possible through judicious applications of adjoint maps to $\mathbf{v}$.

Suppose first that $a_{10} \neq 0$. Scaling $\mathbf{v}$ if necessary, we can assume that $a_{10}=1$. Referring to Table 2 , if we act on such a $\mathbf{v}$ by

$$
\begin{aligned}
\mathbf{v}^{\prime}= & \operatorname{Ad}\left(\exp \left(\frac{a_{8}}{2} \mathbf{v}_{8}\right)\right) \mathbf{v} \\
= & a_{1}^{\prime} \mathbf{v}_{1}+a_{2} \mathbf{v}_{2}+a_{3}^{\prime} \mathbf{v}_{3}+a_{4} \mathbf{v}_{4}+a_{5} \mathbf{v}_{5} \\
& +a_{6}^{\prime} \mathbf{v}_{6}+a_{7} \mathbf{v}_{7}+a_{9} \mathbf{v}_{9}+\mathbf{v}_{10}
\end{aligned}
$$

we can make the coefficient of $a_{8}$ vanish. The remaining onedimensional subalgebras are spanned by vectors of the above form with $a_{10}=0$. If $a_{9} \neq 0$, we scale to make $a_{9}=1$ and then act on $\mathbf{v}$ to cancel the coefficient of $a_{7}$ as follows:

$$
\begin{aligned}
\mathbf{v}^{\prime}= & \operatorname{Ad}\left(\exp \left(\frac{a_{7}}{3} \mathbf{v}_{2}\right)\right) \mathbf{v} \\
= & a_{1}^{\prime} \mathbf{v}_{1}+a_{2} \mathbf{v}_{2}+a_{3}^{\prime} \mathbf{v}_{3}+a_{4} \mathbf{v}_{4} \\
& +a_{5} \mathbf{v}_{5}+a_{6} \mathbf{v}_{6}+a_{8} \mathbf{v}_{8}+\mathbf{v}_{9}
\end{aligned}
$$

We can further act on $\mathbf{v}^{\prime}$ by the group generated by $\mathbf{v}_{4}$; this has the net effect of scaling the coefficients of $\mathbf{v}_{2}$ :

$$
\begin{aligned}
\mathbf{v}^{\prime \prime}= & \operatorname{Ad}\left(\exp \left(\mu \mathbf{v}_{4}\right)\right) \mathbf{v} \\
= & a_{1} \mathbf{v}_{1}+e^{12 \mu} a_{2} \mathbf{v}_{2}+a_{3} \mathbf{v}_{3}+a_{4} \mathbf{v}_{4} \\
& +a_{5} \mathbf{v}_{5}+a_{6} \mathbf{v}_{6}+a_{8} \mathbf{v}_{8}+\mathbf{v}_{9} .
\end{aligned}
$$

So, depending on the sign of $a_{2}$, we can make the coefficient of $\mathbf{v}_{2}$ either $+1,-1$, or 0 . If $a_{10}=a_{9}=0$ and $a_{4} \neq 0$, we scale to make $a_{4}=1$. So, the nonzero vector $\mathbf{v}$ is equivalent to $\mathbf{v}^{\prime}$ under adjoint maps:

$$
\begin{aligned}
\mathbf{v}^{\prime} & =\operatorname{Ad}\left(\exp \left(\frac{a_{7}}{3} \mathbf{v}_{7}\right)\right) \circ \operatorname{Ad}\left(\exp \left(\frac{a_{2}}{12} \mathbf{v}_{2}\right)\right) \mathbf{v} \\
& =a_{1} \mathbf{v}_{1}+a_{3} \mathbf{v}_{3}+\mathbf{v}_{4}+a_{5} \mathbf{v}_{5}+a_{6} \mathbf{v}_{6}+a_{8} \mathbf{v}_{8}
\end{aligned}
$$

If $a_{10}=a_{9}=a_{4}=0$ and $a_{3} \neq 0$, by scaling $\mathbf{v}$, we can assume that $a_{3}=1$. Referring to Table 2 , if we act on such a $\mathbf{v}$ by the following adjoint map, we can arrange that the coefficients of $a_{6}$ vanish:

$$
\begin{aligned}
\mathbf{v}^{\prime}= & \operatorname{Ad}\left(\exp \left(a_{6} \mathbf{v}_{6}\right)\right) \mathbf{v} \\
= & a_{1} \mathbf{v}_{1}+a_{2} \mathbf{v}_{2}+\mathbf{v}_{3}+a_{5} \mathbf{v}_{5} \\
& +a_{6} \mathbf{v}_{6}+a_{7} \mathbf{v}_{7}+a_{8}^{\prime} \mathbf{v}_{8} .
\end{aligned}
$$

If $a_{10}=a_{9}=a_{4}=a_{3}=0$ and $a_{5} \neq 0$, we scale to make $a_{5}=1$. Thus, $\mathbf{v}$ is equivalent to $\mathbf{v}^{\prime}$ under the adjoint representations:

$$
\begin{aligned}
\mathbf{v}^{\prime} & =\operatorname{Ad}\left(\exp \left(\frac{a_{8}}{2} \mathbf{v}_{6}\right)\right) \mathbf{v} \\
& =a_{1} \mathbf{v}_{1}+a_{2} \mathbf{v}_{2}+\mathbf{v}_{5}+a_{6} \mathbf{v}_{6}+a_{7} \mathbf{v}_{7} .
\end{aligned}
$$


If $a_{10}=a_{9}=a_{4}=a_{3}=a_{5}=0$ and $a_{1} \neq 0$, we scale to make $a_{1}=1$. So, we can make the coefficients of $a_{6}, a_{8}$ zero by using the following adjoint maps:

$$
\begin{aligned}
\mathbf{v}^{\prime} & =\operatorname{Ad}\left(\exp \left(-\frac{a_{8}}{2} \mathbf{v}_{10}\right)\right) \circ \operatorname{Ad}\left(\exp \left(-a_{6} \mathbf{v}_{8}\right)\right) \mathbf{v} \\
& =\mathbf{v}_{1}+a_{2} \mathbf{v}_{2}+a_{7} \mathbf{v}_{7} .
\end{aligned}
$$

If $a_{10}=a_{9}=a_{4}=a_{3}=a_{5}=a_{1}=0$ and $a_{2} \neq 0$, by scaling $\mathbf{v}$, we can assume that $a_{2}=1$. Therefore, we can arrange that the coefficients of $a_{7}$ vanish by simplifying the nonzero vector $\mathbf{v}$ as follows:

$$
\begin{aligned}
\mathbf{v}^{\prime} & =\operatorname{Ad}\left(\exp \left(-\frac{a_{7}}{3} \mathbf{v}_{9}\right)\right) \mathbf{v} \\
& =\mathbf{v}_{2}+a_{6} \mathbf{v}_{6}+a_{8} \mathbf{v}_{8}
\end{aligned}
$$

We can further act on $\mathbf{v}^{\prime}$ by the group generated by $\mathbf{v}_{3}$ :

$$
\begin{aligned}
\mathbf{v}^{\prime \prime} & =\operatorname{Ad}\left(\exp \left(\mu \mathbf{v}_{3}\right) \mathbf{v}^{\prime}\right) \\
& =\mathbf{v}_{2}+e^{\mu} a_{6} \mathbf{v}_{6}+a_{8} \mathbf{v}_{8} .
\end{aligned}
$$

So, depending on the sign of $a_{6}$, we can make the coefficient of $\mathbf{v}_{6}$ either $+1,-1$, or 0 . If $a_{10}=a_{9}=a_{4}=a_{3}=a_{5}=a_{1}=a_{2}=0$ and $a_{6} \neq 0$, by scaling $\mathbf{v}$, we can assume that $a_{6}=1$. We can act on such a $\mathbf{v}$ by the group generated by $\mathbf{v}_{4}$. So, depending on the sign of $a_{7}$, we can make the coefficient of $\mathbf{v}_{7}$ either +1 , -1 or 0 . The case $a_{10}=a_{9}=a_{4}=a_{3}=a_{5}=a_{1}=a_{2}=$ $a_{6}=0$ and $a_{7} \neq 0$, no further simplifications are possible. The last remaining case occurs when $a_{10}=a_{9}=a_{4}=$ $a_{3}=a_{5}=a_{1}=a_{2}=a_{4}=a_{6}=a_{7}=0$ and $a_{8} \neq 0$, for which our earlier simplifications were unnecessary. Hence, the only remaining vectors are the multiples of $\mathbf{v}_{8}$, on which the adjoint representation acts trivially.

\section{Approximately Differential Invariants for the Perturbed Harry Dym Equation}

In this section, we compute some approximately differential invariants of the perturbed Harry Dym equation with respect to the optimal system. Consider the operator $\mathbf{v}^{2}$. To determine the independent invariants $I$, we need to solve the first-order partial differential equation:

$$
\left(\varepsilon \frac{\partial}{\partial t}+a \varepsilon x \frac{\partial}{\partial x}+a \varepsilon u \frac{\partial}{\partial u}\right)(I(x, t, u))=0 ;
$$

that is,

$$
\varepsilon \frac{\partial I}{\partial t}+a \varepsilon x \frac{\partial I}{\partial x}+a \varepsilon u \frac{\partial I}{\partial u}=0,
$$

which is a first-order homogeneous PDE. The solution can be found by integrating the corresponding characteristic system of ordinary differential equation, which is

$$
\frac{d x}{a \varepsilon x}=\frac{d t}{\varepsilon}=\frac{d u}{a \varepsilon u} \text {. }
$$

TABLE 3: Approximately differential invariants for the perturbed Harry Dym equation.

\begin{tabular}{lc}
\hline Operator & Approximate differential invariants \\
\hline $\mathbf{v}_{1}$ & $x, u$ \\
$\mathbf{v}_{2}$ & $t, \frac{u}{x}$ \\
$\mathbf{v}_{3}$ & $x, u t^{1 / 3}$ \\
$\mathbf{v}_{4}$ & $t, \frac{u}{x^{2}}$ \\
$\mathbf{v}_{5}$ & $-\frac{\ln x}{a}+t, \frac{u}{x}$ \\
$\mathbf{v}_{7}+a \mathbf{v}_{8}$ & $t, \frac{u}{x+1}$ \\
$\mathbf{v}_{6}+\mathbf{v}_{8}$ & $-\ln (x+1)+t, \frac{u}{x+1}$ \\
$\mathbf{v}_{6}-\mathbf{v}_{7}+\mathbf{v}_{8}$ & $-\frac{\ln (x+1)+t, \frac{u}{x+1}}{a \varepsilon}+t, \frac{u}{x}$ \\
$\mathbf{v}_{6}+\mathbf{v}_{7}+\mathbf{v}_{8}$ & $-\frac{\ln (a x-1)}{a \varepsilon}+t, \frac{u}{a x-1}$ \\
$\mathbf{v}_{2}+a \mathbf{v}_{8}$ & $-\frac{\ln (a x+1)}{a \varepsilon}+t, \frac{u}{a x+1}$ \\
$\mathbf{v}_{2}-\mathbf{v}_{6}+a \mathbf{v}_{8}$ & $-b \varepsilon x-a \varepsilon+t, u$ \\
$\mathbf{v}_{2}+\mathbf{v}_{6}+a \mathbf{v}_{8}$ & $\frac{-d \varepsilon-b}{\sqrt{c \varepsilon+a}}$ \\
$\mathbf{v}_{1}+a \mathbf{v}_{2}+b \mathbf{v}_{7}$ & $\arctan \left(\frac{x}{\sqrt{c \varepsilon+a}}\right)+t, \frac{u}{x^{2}+c \varepsilon+a}$ \\
$a \mathbf{v}_{1}+b \mathbf{v}_{2}+\mathbf{v}_{5}$ & $+c \mathbf{v}_{6}+d \mathbf{v}_{7}$
\end{tabular}

Hence, the independent approximately differential invariants are as follows:

$$
y=\frac{u}{x}, \quad v=\frac{\ln x-a t}{a} .
$$

In this manner, we investigate some independent approximately differential invariants with respect to the optimal system which are listed in Table 3.

\section{Conclusions}

In this paper, we investigate the approximate symmetry of the perturbed Harry Dym equation and discuss the structure of its Lie algebra. Moreover, we compute optimal system of onedimensional approximate Lie algebras of the perturbed Harry Dym equation and derive some approximately differential invariants with respect to the generators of Lie algebra and optimal system.

\section{References}

[1] W. Hereman, P. P. Banerjee, and M. R. Chatterjee, "Derivation and implicit solution of the Harry Dym equation and its connections with the Korteweg-de Vries equation," Journal of Physics A, vol. 22, no. 3, pp. 241-255, 1989.

[2] M. D. Kruskal, Lecture Notes in Physics, vol. 38, Springer, Berlin, Germany, 1975.

[3] P. C. Sabatier, "On some spectral problems and isospectral evolutions connected with the classical string problem-II: evolution equation," Lettere Al Nuovo Cimento, vol. 26, no. 15, pp. 483-486, 1979. 
[4] L. Yi-Shen, "Evolution equations associated with the eigenvalue problem based on the equation $\varphi_{x x}=\left[v(x)-\kappa^{2} \rho^{2}(x)\right] \varphi$," Lettere Al Nuovo Cimento, vol. 70, no. 1, pp. 1-12, 1982.

[5] Z. J. Qiao, "A completely integrable system associated with the Harry Dym hierarchy," Journal of Nonlinear Mathematical Physics, vol. 1, no. 1, pp. 65-74, 1994.

[6] Z. Qiao, "Commutator representations of nonlinear evolution equations: Harry Dym and Kaup-Newell cases," Journal of Nonlinear Mathematical Physics, vol. 2, no. 2, pp. 151-157, 1995.

[7] F. Calogero and A. Degasperis, Spectral Transform and Solitons, vol. 1, North-Holland, Amsterdam, The Netherlands, 1982.

[8] M. Wadati, Y. H. Ichikawa, and T. Shimizu, "Cusp soliton of a new integrable nonlinear evolution equation," Progress of Theoretical Physics, vol. 64, no. 6, pp. 1959-1967, 1980.

[9] M. Wadati, K. Konno, and Y. H. Ichikawa, "New integrable nonlinear evolution equations," Journal of the Physical Society of Japan, vol. 47, no. 5, pp. 1698-1700, 1979.

[10] F. Magri, "A simple model of the integrable Hamiltonian equation," Journal of Mathematical Physics, vol. 19, no. 5, pp. 1156-1162, 1978.

[11] P. J. Olver, Application of Lie Groups To Differential Equations, Springer, New York, NY, USA, 2nd edition, 1993.

[12] V. A. Baikov, R. K. Gazizov, and N. Kh. Ibragimov, "Approximate symmetries of equations with a small parameter," Matematicheskiu Sbornik, vol. 136, no. 4, pp. 435-450, 1988, English Translation in Mathematics of the USSR, vol. 64, pp. 427-441, 1989.

[13] V. A. Baikov, R. K. Gazizov, and N. H. Ibragimov, "Approximate transformation groups and deformations of symmetry Lie algebras," in CRC Handbook of Lie Group Analysis of Differential Equation, N. H. Ibragimov, Ed., vol. 3, chapter 2, CRC Press, Boca Raton, Fla, USA, 1996.

[14] W. I. Fushchich and W. M. Shtelen, "On approximate symmetry and approximate solutions of the nonlinear wave equation with a small parameter," Journal of Physics A, vol. 22, no. 18, pp. L887L890, 1989.

[15] N. Euler, M. W. Shulga, and W.-H. Steeb, "Approximate symmetries and approximate solutions for a multidimensional LandauGinzburg equation," Journal of Physics A, vol. 25, no. 18, pp. L1095-L1103, 1992.

[16] M. Euler, N. Euler, and A. Kohler, "On the construction of approximate solutions for a multidimensional nonlinear heat equation," Journal of Physics A, vol. 27, no. 6, pp. 2083-2092, 1994.

[17] M. Pakdemirli, M. Yürüsoy, and I. T. Dolapçi, "Comparison of approximate symmetry methods for differential equations," Acta Applicandae Mathematicae, vol. 80, no. 3, pp. 243-271, 2004.

[18] R. Wiltshire, "Two approaches to the calculation of approximate symmetry exemplified using a system of advection-diffusion equations," Journal of Computational and Applied Mathematics, vol. 197, no. 2, pp. 287-301, 2006.

[19] N. H. Ibragimov and V. F. Kovalev, Approximate and Renormgroup Symmetries, Nonlinear Physical Science, Higher Education Press, Beijing, China, 2009, A. C.J Luo and N.H Ibragimov, Eds.

[20] J. Patera, R. T. Sharp, P. Winternitz, and H. Zassenhaus, "Invariants of real low dimension Lie algebras," Journal of Mathematical Physics, vol. 17, no. 6, pp. 986-994, 1975. 


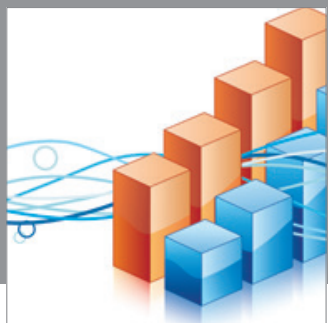

Advances in

Operations Research

mansans

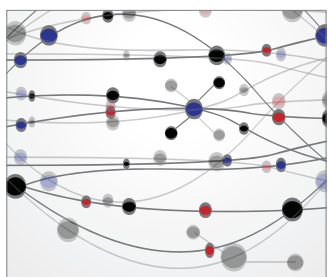

The Scientific World Journal
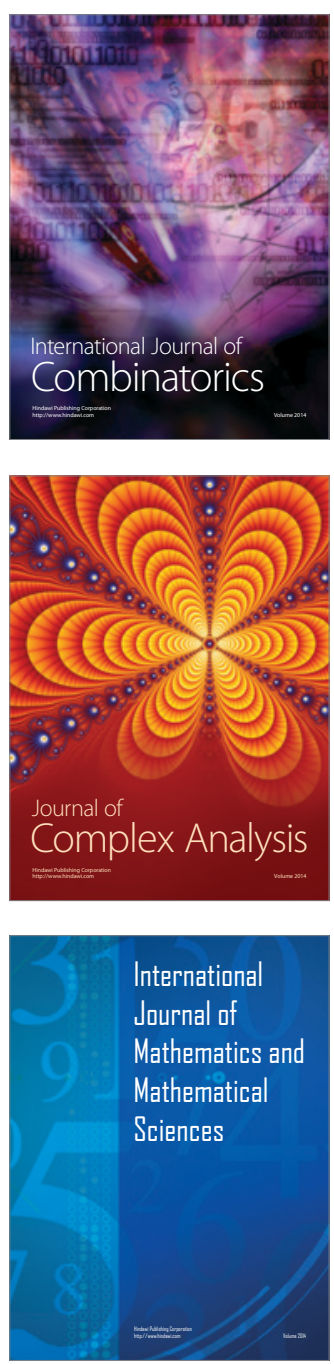
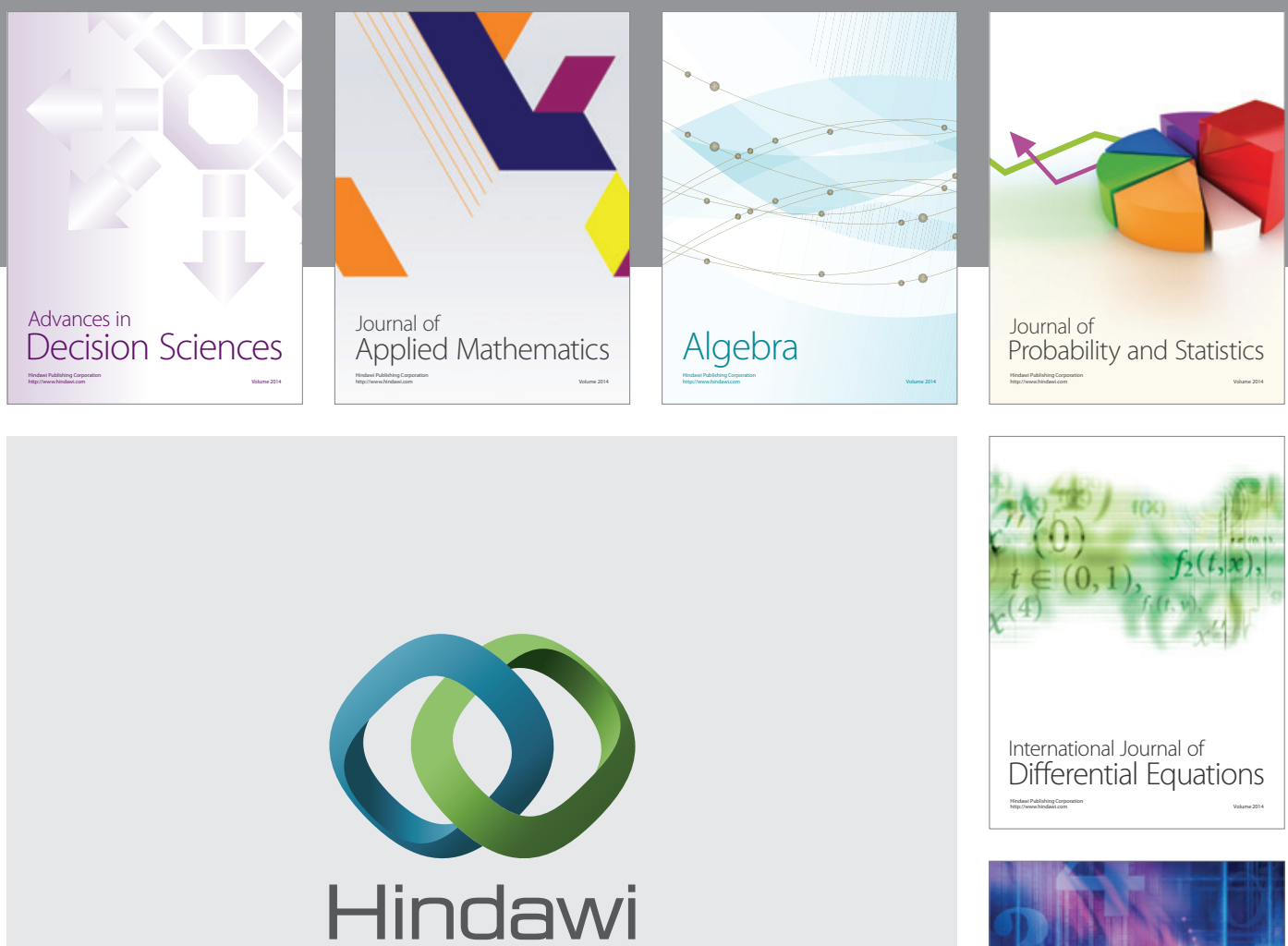

Submit your manuscripts at http://www.hindawi.com
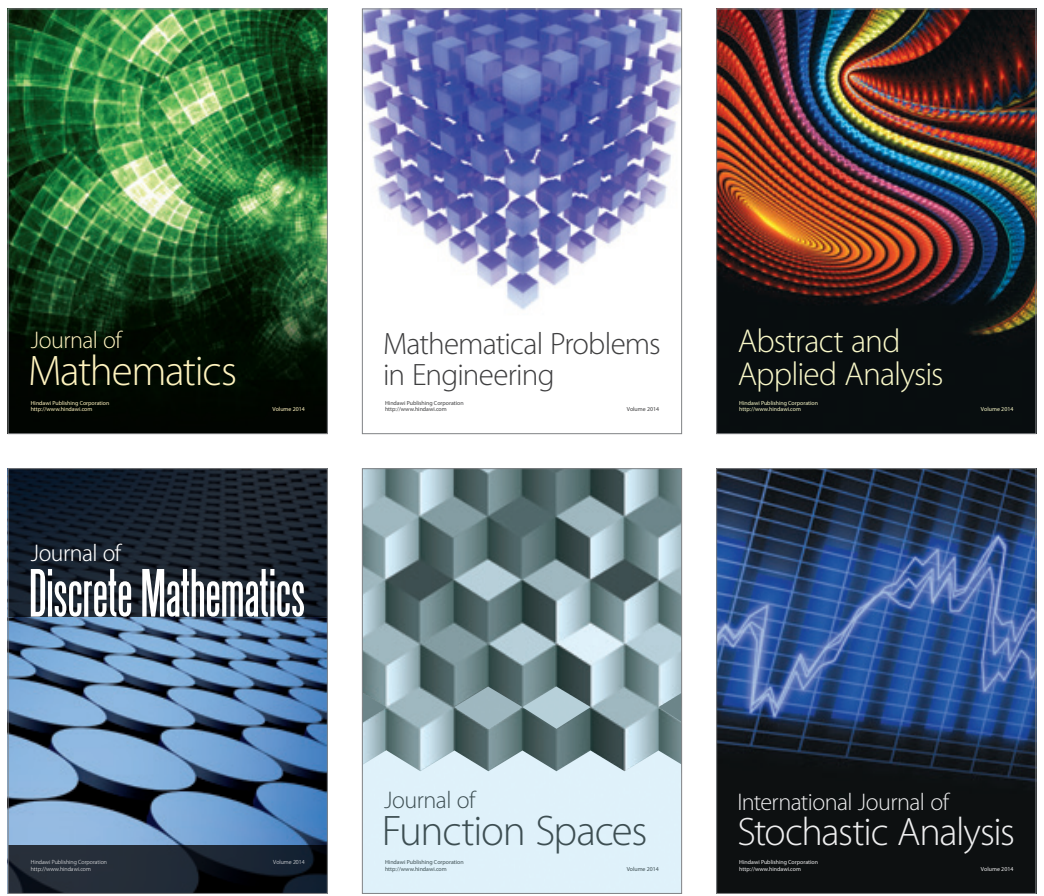

Journal of

Function Spaces

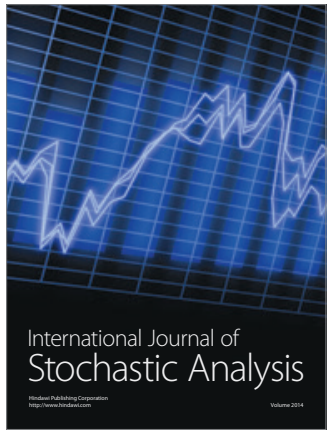

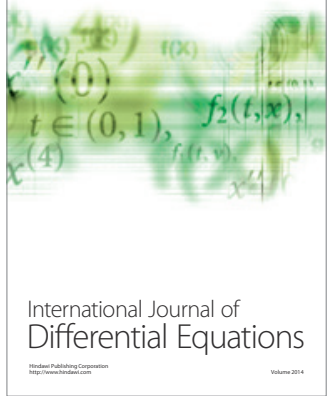
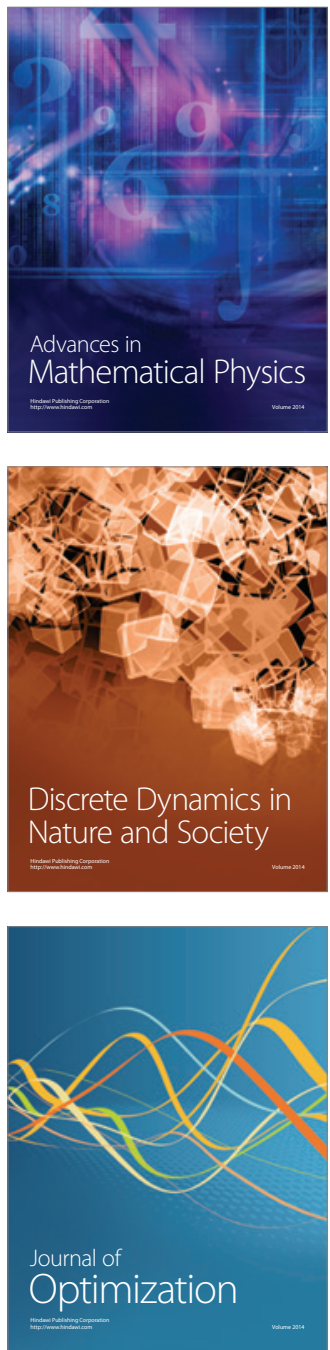